\title{
Wide Range Temperature Sensors Based on One-Dimensional Photonic Crystal with a Single Defect
}

\author{
Arun Kumar, ${ }^{1}$ Vipin Kumar, ${ }^{2}$ B. Suthar, ${ }^{3}$ A. Bhargava, ${ }^{4}$ Kh. S. Singh, ${ }^{2}$ and S. P. Ojha ${ }^{5}$ \\ ${ }^{1}$ AITTM, Amity University, NOIDA 201303, India \\ ${ }^{2}$ Department of Physics, Digamber Jain (P.G.) College, Baraut 250611, India \\ ${ }^{3}$ Department of Physics, Govt. College of Engineering \& Technology, Bikaner 334004, India \\ ${ }^{4}$ Nanophysics Laboratory, Department of Physics, Govt. Dungar College, Bikaner 334001, India \\ ${ }^{5}$ Director General, IIMT Group of Colleges, Noida 201303, India \\ Correspondence should be addressed to Vipin Kumar, vrpcommon@gmail.com
}

Received 21 March 2012; Revised 9 June 2012; Accepted 27 June 2012

Academic Editor: Yeou Song (Brian) Lee

Copyright ( $) 2012$ Arun Kumar et al. This is an open access article distributed under the Creative Commons Attribution License, which permits unrestricted use, distribution, and reproduction in any medium, provided the original work is properly cited.

\begin{abstract}
Transmission characteristics of one-dimensional photonic crystal structure with a defect have been studied. Transfer matrix method has been employed to find the transmission spectra of the proposed structure. We consider a Si/air multilayer system and refractive index of Si layer has been taken as temperature dependent. As the refractive index of Si layer is a function of temperature of medium, so the central wavelength of the defect mode is a function of temperature. Variation in temperature causes the shifting of defect modes. It is found that the average change or shift in central wavelength of defect modes is $0.064 \mathrm{~nm} / \mathrm{K}$. This property can be exploited in the design of a temperature sensor.
\end{abstract}

\section{Introduction}

Since the last two and half decades, investigations on various properties of photonic crystals, particularly photonic bandgap materials, have become an area of interest for many researchers [1-6]. It was observed that periodic modulation of the dielectric functions significantly modifies the spectral properties of the electromagnetic waves. The transmission and reflection spectra of such structures are characterized by the presence of allowed and forbidden photonic bands bands similar to the electronic band structure of periodic potentials. For this reason, such a new class of artificial optical material with periodic dielectric modulation is known as photonic bandgap (PBG) material [3]. Fundamental optical properties like band structure, reflectance, group velocity and the rate of spontaneous emission, and so forth can be controlled effectively by changing the spatial distribution of the dielectric function $[4,5]$. This fact has opened up important possibilities for the design of novel optical and optoelectronic devices. Conventional photonic crystals have periodic modulation of homogeneous refractive indices, and they are artificially fabricated with periods that are comparable to the wavelength of the electromagnetic waves. These photonic crystals lead to formation of photonic bandgaps or stop bands, in which propagation of electromagnetic waves of certain wavelengths is prohibited. A onedimensional photonic crystal (1D PC) structure has many interesting applications such as dielectric reflecting mirrors, optical switches, filters, and optical limiters. It has also been demonstrated theoretically and experimentally that 1D PCs can have absolute omnidirectional PBGs [7-11].

In addition to the existence of wide bandgaps in some properly designed PCs, the feature of a tunable PBG is an interesting property of such PCs. The PBG can be tuned by means of some external agents [12]. For instance, it can be changed by the operating temperature and we call it T-tuning. A superconductor/dielectric PC belongs to this class of PC. This happens because of the temperaturedependent London perturbation length in the superconducting materials [13-15]. Using a liquid crystal as one of the constituents in a PC, the T-tuning optical response is also obtainable [16]. Recently, PCs containing semiconductor as 
one of the constituents have also been investigated by many researchers. PCs with intrinsic semiconductor belong to $\mathrm{T}$ tuning devices because the dielectric constant of an intrinsic semiconductor is strongly dependent on temperature [17].

Although these applications can be realized using pure PCs, but doped or defective PCs may be more useful, just as semiconductor doped by impurities is more important than the pure ones for various applications. The idea of doped PCs comes from the consideration of the analogy between electromagnetism and solid state physics, which lead to the study of band structures of periodic materials and further to the possibility of the occurrence of localized modes in the bandgap when a defect is introduced in the lattice. These defect-enhanced structures are called doped photonic crystals and present some resonant transmittance peaks in the bandgap corresponding to the occurrence of the localized states [18], due to the change of the interference behavior of the incident waves. Defect(s) can be introduced into perfect PCs by changing the thickness of the layer [19], inserting another dielectric into the structure [20], or removing a layer from it $[21,22]$.

The introduction of the defect states within PCs has been perceived as a new dimension in the study of photonic crystals, especially in 2D and 3D PCs, due to numerous possible applications that can be achieved by using them. In $2 \mathrm{D}$ or $3 \mathrm{D}$ PCs, it has been known that a point defect can act as a micro cavity, a line defect like a waveguide, and a planar defect like a perfect mirror [6]. Similar to 2D or 3D PCs, the introduction of the defect layers in 1D PCs can also create localized defect modes within the PBGs. Due to the simplicity in $1 \mathrm{D}$ PC fabrications over $2 \mathrm{D}$ and $3 \mathrm{D}$ PCs, the defect mode can be easily introduced within1D PCs. Such PCs with defect(s) can be exploited for the design of various applications such as in the design of TE/TM filters and splitters [23], in the fabrication of lasers [24], and in light emitting diodes [25]. The existence of defect mode in 1D PCs depends upon a number of parameters such as refractive indices of the layers, filling fraction, the thickness of defect layer, and the angle of incidence. If all other parameters are kept constant and the refractive index of the material is changed, then any change in refractive index of a material results to a change or shift the position of the defect mode in the reflection/transmission spectra. In this present communication, a wide range temperature sensor based on one-dimensional photonic crystal with a single defect has been proposed.

Here, we consider the Si/air multilayer system with a defect in the form of a single Si layer in the middle. Si is taken as one of the constituents of a one-dimensional photonic crystal. Since the refractive index of Si depends on temperature [17], so any change in the temperature of the constituent material will change the refractive index of the material, and hence the wavelength corresponding to the middle of the defect mode will also change or shift. By measuring this change or shift in the position of defect mode, the change in the temperature can be measured, so it can work as a temperature sensor. In this present work, we will restrict our study for normal incidence only.

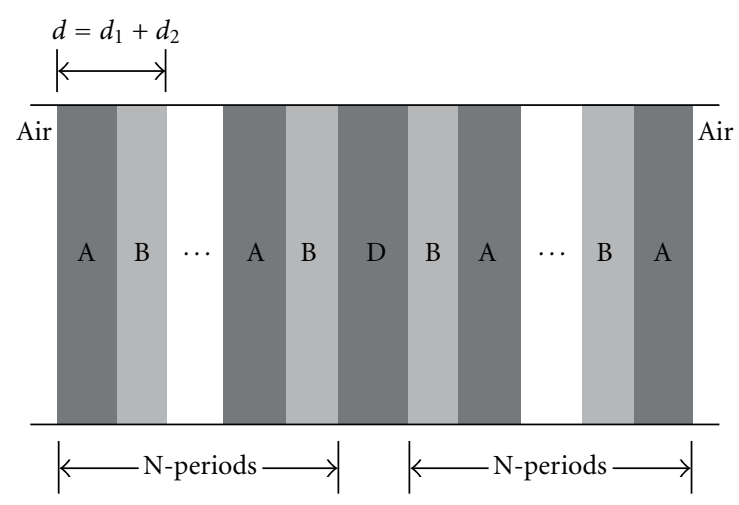

FIGURE 1: Schematic diagram of 1-D photonic crystal with defect.

\section{Theoretical Analysis}

The schematic representation of one-dimensional photonic crystal with defect is represented in Figure 1.

We consider air/ $(\mathrm{AB})^{N} \mathrm{D}(\mathrm{BA})^{N} /$ air, in which $\mathrm{A}$ and $B$ represent the layers of high and low refractive index materials, and D is the defect layer. To compute the defect mode in the transmission spectrum, we employ the transfer matrix method (TMM) $[26,27]$. In this method, the transfer matrix for each layer can be written as

$$
M_{j}=Z_{j} P_{j} Z_{j}^{-1}
$$

where $j$ stands for $\mathrm{A}, \mathrm{B}$, or $\mathrm{D}$ layers and $Z_{j}$ and $P_{j}$ are called the dynamical matrix, and the propagation matrix, respectively. The dynamical matrix is given by the following equations:

$Z_{j}=\left(\begin{array}{cc}1 & 1 \\ n_{j} \cos \theta_{j} & -n_{j} \cos \theta_{j}\end{array}\right)$, for TE mode of polarization, $Z_{j}=\left(\begin{array}{cc}\cos \theta_{j} & \cos \theta_{j} \\ n_{j} & -n_{j}\end{array}\right)$, for TM mode of polarization,

where $\theta_{j}$ is the ray angle in the layers and $n_{j}$ is the refractive index of the layers. Also, the propagation matrix $P_{j}$ can be defined as

$$
P_{j}=\left(\begin{array}{cc}
e^{i \delta_{j}} & 0 \\
0 & e^{-i \delta_{j}}
\end{array}\right)
$$

where the phase is written as

$$
\delta_{j}=\frac{2 \pi d_{j}}{\lambda} n_{j} \cos \theta_{j}
$$

The transfer matrix, for the structure (Figure 1), embedded in air, air/ $(\mathrm{AB})^{N} \mathrm{D}(\mathrm{BA})^{N} /$ air can be written as

$$
M=\left(\begin{array}{ll}
M_{11} & M_{12} \\
M_{21} & M_{22}
\end{array}\right)=Z_{0}^{-1}\left(M_{\mathrm{A}} M_{\mathrm{B}}\right)^{N} M_{\mathrm{D}}\left(M_{\mathrm{A}} M_{\mathrm{B}}\right)^{N} Z_{0},
$$

where $Z_{0}$ is called the dynamical matrix for air. 
The transmittance $(T)$ and reflectance $(R)$ can be calculated by using the matrix elements $M_{11}$ and $M_{21}$ given in (5) as follows:

$$
T=\left|\frac{1}{M_{11}}\right|^{2}, \quad R=\left|\frac{M_{21}}{M_{11}}\right|^{2} .
$$

In the next section, structural parameters of the device have been defined.

\section{Proposed Structure and Structural Parameters}

We choose Si for the materials A and D, air for material B, and $N=5$ in Figure 1. Silicon and air have been taken as the high and the low refractive index materials, respectively, and an additional silicon layer (D) as the defect layer. The geometrical parameters are so chosen that the thicknesses of high and low refractive index materials are $d_{1}=600 \mathrm{~nm}, d_{2}=$ $1200 \mathrm{~nm}$ and the thickness of defect layer is taken $1800 \mathrm{~nm}$. The thermal expansion coefficient for silicon layer is $2.6 \times$ $10^{-6} / \mathrm{K}$, which is negligible small quantity. So we neglect the thermal expansion of Si layer in our computation.

The refractive index of air is independent of temperature and wavelength. But the refractive index of silicon layer is taken as a function of both wavelength and temperature. The refractive index of Silicon ( $\mathrm{Si}$ ) in the ranges 1.2 to $14 \mu \mathrm{m}$ and $20-1600 \mathrm{~K}$ is represented as [17]

$$
\begin{aligned}
n^{2}(\lambda, T)= & \varepsilon(T)+\frac{e^{-3 \Delta L(T) / L_{293}}}{\lambda^{2}} \\
& \times\left(0.8948+4.3977 \times 10^{-4} T+7.3835 \times 10^{-8} T^{2}\right),
\end{aligned}
$$

where

$$
\begin{aligned}
\mathcal{E}(T)= & 11.4445+2.7739 \times 10^{-4} T+1.7050 \\
& \times 10^{-6} T^{2}-8.1347 \times 10^{-10} T^{3} \\
\frac{\Delta L(T)}{L_{293}}= & -0.071+1.887 \times 10^{-6} T+1.934 \times 10^{-9} T^{2} \\
& -4.554 \times 10^{-13} T^{3} \quad \text { for } 293 \mathrm{~K} \leq T \leq 1600 \mathrm{~K}, \\
\frac{\Delta L(T)}{L_{293}}= & -0.021-4.149 \times 10^{-7} T-4.620 \times 10^{-10} T^{2} \\
& +1.482 \times 10^{-11} T^{3} \quad \text { for } 20 \mathrm{~K} \leq T \leq 293 \mathrm{~K} .
\end{aligned}
$$

The plot of the refractive index as the function of wavelength and temperature is shown in Figure 2. From Figure 2, it is clear that the refractive index of silicon layer increases with the increase in temperature. Therefore, the refractive index contrast increases with temperature.

In the next section, we will compute the transmission spectra of photonic crystal with defect by using (6) as shown in Figure 1.

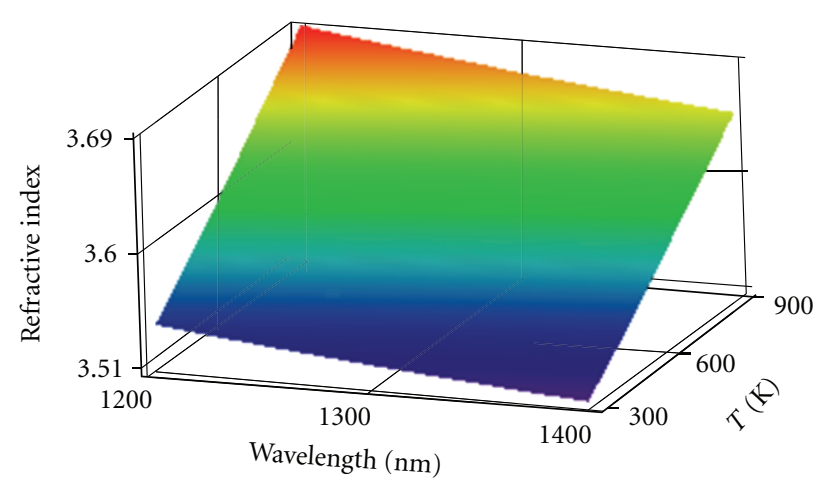

FIGURE 2: Variation of refractive index of $\mathrm{Si}$ as a function of wavelength and temperature.

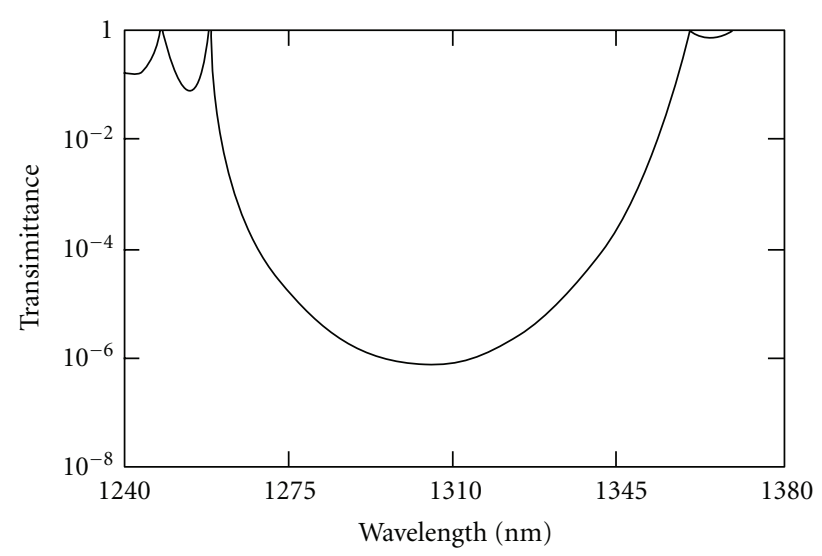

Figure 3: Transmittance for an ideal PC of (Si/air) ${ }^{10}$ at $100 \mathrm{~K}$ temperature.

\section{Result and Discussion}

In this section, we present the working principle of the proposed temperature sensor. The transmission spectra of the ideal PC (Si/air) ${ }^{10}$ at $100 \mathrm{~K}$ temperature for normal incidence are shown in Figure 3. If a defect which is in the form of silicon layer is introduced in this PC, then a defect mode appears inside the bandgap. The transmission spectra of the PC with a defect $\left[(\mathrm{Si} / \mathrm{air})^{5} \mathrm{Si}(\mathrm{air} / \mathrm{Si})^{5}\right]$ at $100 \mathrm{~K}$ temperature are shown in Figure 4. As shown in this figure a defect mode has a mid-wavelength centered at $1288.3 \mathrm{~nm}$. Thus, from this figure it is clear that if the incident radiation of wavelengths ranging from $1275 \mathrm{~nm}$ to $1350 \mathrm{~nm}$ is incident normally on the proposed structure at $100 \mathrm{~K}$, then it will pass only $1288.3 \mathrm{~nm}$ wavelength and all other wavelengths will be reflected. More interestingly, this defect mode can be shifted to the longer wavelength region by the variation of temperature. The transmission spectra of the proposed structure at various temperature are shown in Figure 5, and the corresponding data is tabulated in Table 1. From Figure 5 and Table 1, it is clear that the transmission peak centered at $1288.3 \mathrm{~nm}$ at $100 \mathrm{~K}$ has been shifted to $1300.08 \mathrm{~nm}$, $1312.9 \mathrm{~nm}$, and $1326.8 \mathrm{~nm}$ corresponding to temperatures at $300 \mathrm{~K}, 500 \mathrm{~K}$, and $700 \mathrm{~K}$, respectively. Further, it is also clear that as the temperature of the structure increases, 


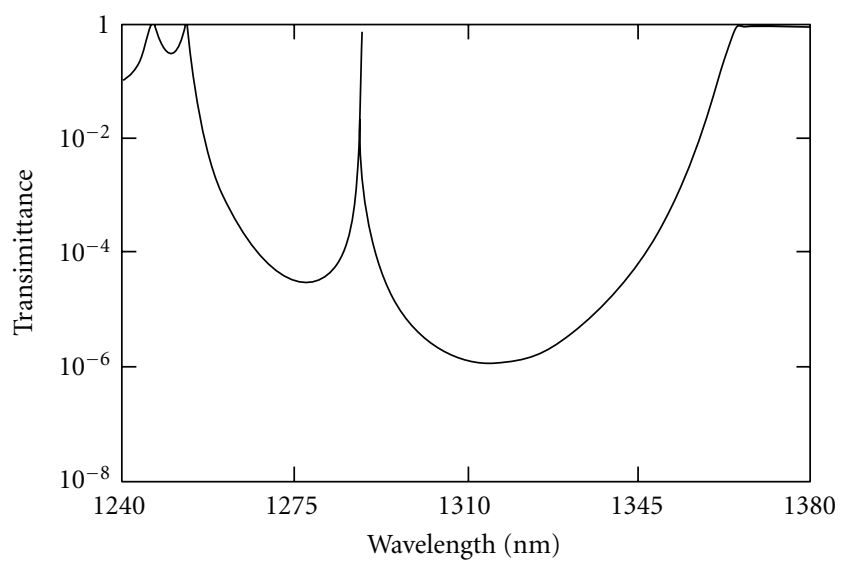

Figure 4: The defect mode for a PC with defect of $\left[(\mathrm{Si} / \text { air })^{5} \mathrm{Si}(\text { air } / \mathrm{Si})^{5}\right]$ at $100 \mathrm{~K}$ temperature.

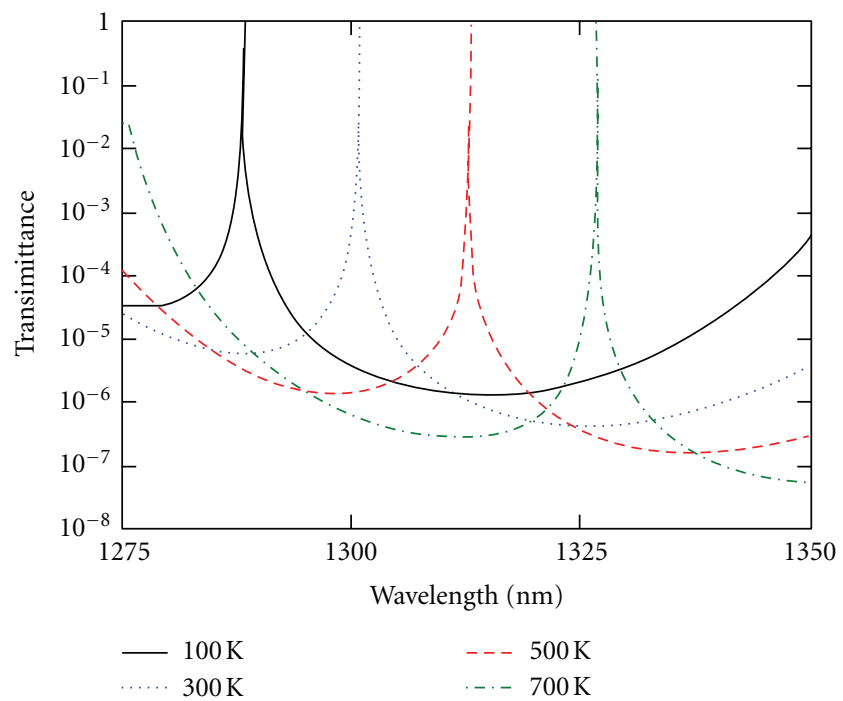

FIGURE 5: Transmission spectra of proposed structure at different temperature.

the defect mode of transmission shifts towards the higher wavelength region. The dependence of defect modes on temperature is due to the dependence of refractive index on temperature as given in (7). The shifting behavior can be explained by using the phase relation given by (4). According to this equation, as refractive index $n(\lambda, T)$ increases with temperature, the wavelength must increase accordingly to keep the phase $\delta$ unchanged. The variation of central wavelength of defect modes with temperature is shown in Figure 6. It is found that the central wavelength of defect modes changes approximately linearly with temperature. The average change in central wavelength of defect mode is $0.064 \mathrm{~nm} / \mathrm{K}$. This change in central wavelength is almost 9 times more than the previous reported work [28]. So the proposed structure is more sensitive to temperature variation. Earlier studies on defect filters reported response time of the order of $\sim \mathrm{ms}$ to $\sim$ ps range with different techniques $[29,30]$. Gan et al. achieved a response rise time

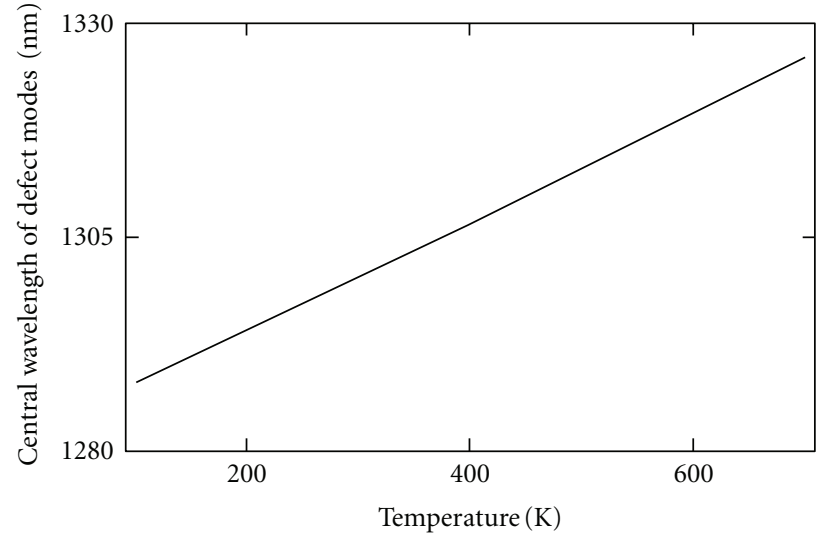

Figure 6: The variation of central wavelength of defect modes with temperature.

TABle 1: Central wavelength of defect modes.

\begin{tabular}{lc}
\hline Temperature $(\mathrm{K})$ & Central wavelength of defect mode $(\mathrm{nm})$ \\
\hline 100 & 1288.3 \\
300 & 1300.8 \\
500 & 1312.9 \\
700 & 1326.8 \\
\hline
\end{tabular}

of $7 \mu$ s and a fall time of $14 \mu \mathrm{s}$ for silicon photonic structures [31]. For the proposed temperature sensor, the response time for silicon will decide slow or fast response. Thus, by measuring the change or shift in the central wavelength of the defect mode, we can measure the temperature in a wide range. Hence, the proposed structure may be used as a temperature sensor in a wide range.

\section{Conclusion}

In the present communication, a simple design of temperature sensor has been proposed. The proposed structure is based on the one-dimensional photonic structure with defect. It is found that introduction of defect in the conventional one-dimensional photonic crystal creates a defect mode. Silicon has been chosen as one of the constituents of the device, because refractive index of the Si depends on the temperature. It is found that centre of this defect mode depends on the temperature of the device and it change linearly with temperature. An average change or shift in the central wavelength of defect mode is $0.064 \mathrm{~nm} / \mathrm{K}$. So, we can use the proposed device as a temperature sensor. The proposed device may also have many applications in different optical systems.

\section{References}

[1] S. John, "Strong localization of photons in certain disordered dielectric superlattices," Physical Review Letters, vol. 58, no. 23, pp. 2486-2489, 1987.

[2] E. Yablonovitch, "Inhibited spontaneous emission in solidstate physics and electronics," Physical Review Letters, vol. 58, no. 20, pp. 2059-2062, 1987. 
[3] C. Soukoulis, Photonic Band Gap Materials, Kluwer Academic, Dordrecht, The Netherlands, 1996.

[4] J. A. M. Rojas, J. Alpuente, J. Piñeiro, and R. Sánchez, "Rigorous full vectorial analysis of electromagnetic wave propagation in ID inhomogeneous media," Progress in Electromagnetics Research, vol. 63, pp. 89-105, 2006.

[5] E. Yablonovitch and T. J. Gmitter, "Photonic band structure: the face-centered-cubic case," Physical Review Letters, vol. 63, no. 18, pp. 1950-1953, 1989.

[6] H. Y. Lee and T. Yao, "Design and evaluation of omnidirectional one-dimensional photonic crystals," Journal of Applied Physics, vol. 93, no. 2, pp. 819-830, 2003.

[7] J. P. Dowling, "Mirror on the wall: you're omnidirectional after all?” Science, vol. 282, no. 5395, pp. 1841-1842, 1998.

[8] E. Yablonovitch, "Engineered omnidirectional externalreflectivity spectra from one-dimensional layered interference filters," Optics Letters, vol. 23, no. 21, pp. 1648-1649, 1998.

[9] D. N. Chigrin, A. V. Lavrinenko, D. A. Yarotsky, and S. V. Gaponenko, "Observation of total omnidirectional reflection from a one-dimensional dielectric lattice," Applied Physics A, vol. 68, no. 1, pp. 25-28, 1999.

[10] B. Suthar, V. Kumar, Kh. S. Singh, and A. Bhargava, "Tuning of photonic band gaps in one dimensional chalcogenide based photonic crystal," Optics Communications, vol. 285, no. 6, pp. 1505-1509, 2012.

[11] V. Kumar, Kh. S. Singh, S. K. Singh, and S. P. Ojha, "Broadening of omnidirectional photonic band gap in Si-based one dimensional photonic crystals," Progress in Electromagnetics Research M, vol. 14, pp. 101-111, 2010.

[12] B. Suthar, V. Kumar, A. Kumar, Kh. S. Singh, and A. Bhargava, "Thermal expansion of photonic band gap for one dimensional photonic crystal," Progress in Electromagnetics Research Letters, vol. 32, pp. 81-90, 2012.

[13] O. L. Berman, Y. E. Lozovik, S. L. Eiderman, and R. D. Coalson, "Superconducting photonic crystals: numerical calculations of the band structure," Physical Review B, vol. 74, no. 9, Article ID 092505, 2006.

[14] H. Takeda and K. Yoshino, "Tunable photonic band schemes in two-dimensional photonic crystals composed of copper oxide high-temperature superconductors," Physical Review B, vol. 67, no. 24, Article ID 245109, 6 pages, 2003.

[15] W. H. Lin, C. J. Wu, T. J. Yang, and S. J. Chang, "Terahertz multichanneled filter in a superconducting photonic crystal," Optics Express, vol. 18, no. 26, pp. 27155-27166, 2010.

[16] P. Halevi, J. A. Reyes-Avendaño, and J. A. Reyes-Cervantes, "Electrically tuned phase transition and band structure in a liquid-crystal-infilled photonic crystal," Physical Review E, vol. 73, no. 4, Article ID 040701(R), 4 pages, 2006.

[17] H. H. Li, "Refractive index of silicon and germanium and its wavelength and temperature derivatives," The Journal of Physical Chemistry, vol. 9, no. 3, pp. 561-658, 1980.

[18] G. Guida, A. de Lustrac, and P. Priou, "An introduction to Photonic Band Gap (PBG) materials," Progress in Electromagnetics Research Letters, vol. 41, pp. 1-20, 2003.

[19] B. Suthar and A. Bhargava, "Temperature-dependent tunable photonic channel filter," IEEE Photonics Technology Letters, vol. 24, no. 5, Article ID 6096365, pp. 338-340, 2012.

[20] C. M. Soukoulis, "Photonic band gaps and localization," in Proceedings of the NATO Advanced Research Workshop, Plenum Press, London, UK, May 1993.

[21] D. R. Smith, R. Dalichaouch, N. Kroll, S. Schultz, S. L. McCall, and P. M. Platzman, "Photonic band structure and defects in one and two dimensions," Journal of the Optical Society of America B, vol. 10, no. 2, pp. 314-321, 1993.
[22] V. Kumar, Kh. S. Singh, and S. P. Ojha, "Abnormal behaviour of one-dimensional photonic crystal with defect," Optik, vol. 122, no. 13, pp. 1183-1187, 2011.

[23] Z. M. Jiang, B. Shi, D. T. Zhao, J. Liu, and X. Wang, "Siliconbased photonic crystal heterostructure," Applied Physics Letters, vol. 79, no. 21, pp. 3395-3397, 2001.

[24] W. D. Zhou, J. Sabarinathan, P. Bhattarcharya et al., "Characteristics of a photonic bandgap single defect microcavity electroluminescent device," IEEE Journal of Quantum Electronics, vol. 37, no. 9, pp. 1153-1160, 2001.

[25] M. W. Feise, I. V. Shadrivov, and Y. S. Kivshar, "Bistable diode action in left-handed periodic structures," Physical Review E, vol. 71, no. 3, Article ID 037602, 4 pages, 2005.

[26] P. Yeh, Optical Waves in Layered Media, John Wiley and Sons, New York, NY, USA, 1988.

[27] M. Born and E. Wolf, Principle of Optics, Pergamon, Oxford, UK, 4th edition, 1970.

[28] W. C. L. Hopman, P. Pottier, D. Yudistira et al., "Quasi-onedimensional photonic crystal as a compact building-block for refractometric optical sensors," IEEE Journal on Selected Topics in Quantum Electronics, vol. 11, no. 1, pp. 11-16, 2005.

[29] S. Toyoda, N. Ooba, A. Kaneko, M. Hikita, T. Kurihara, and T. Maruno, "Wideband polymer thermo-optic wavelength tunable filter with fast response for WDM systems," Electronics Letters, vol. 36, no. 7, pp. 658-660, 2000.

[30] X. Hu, Q. Gong, Y. Liu, B. Cheng, and D. Zhang, "Ultrafast tunable filter in two-dimensional organic photonic crystal," Optics Letters, vol. 31, no. 3, pp. 371-373, 2006.

[31] F. Gan, T. Barwicz, M. A. Popović et al., "Maximizing the thermo-optic tuning range of silicon photonic structures," in Proceeding of the IEEE International Conference on Photonics in Switching, August 2007. 

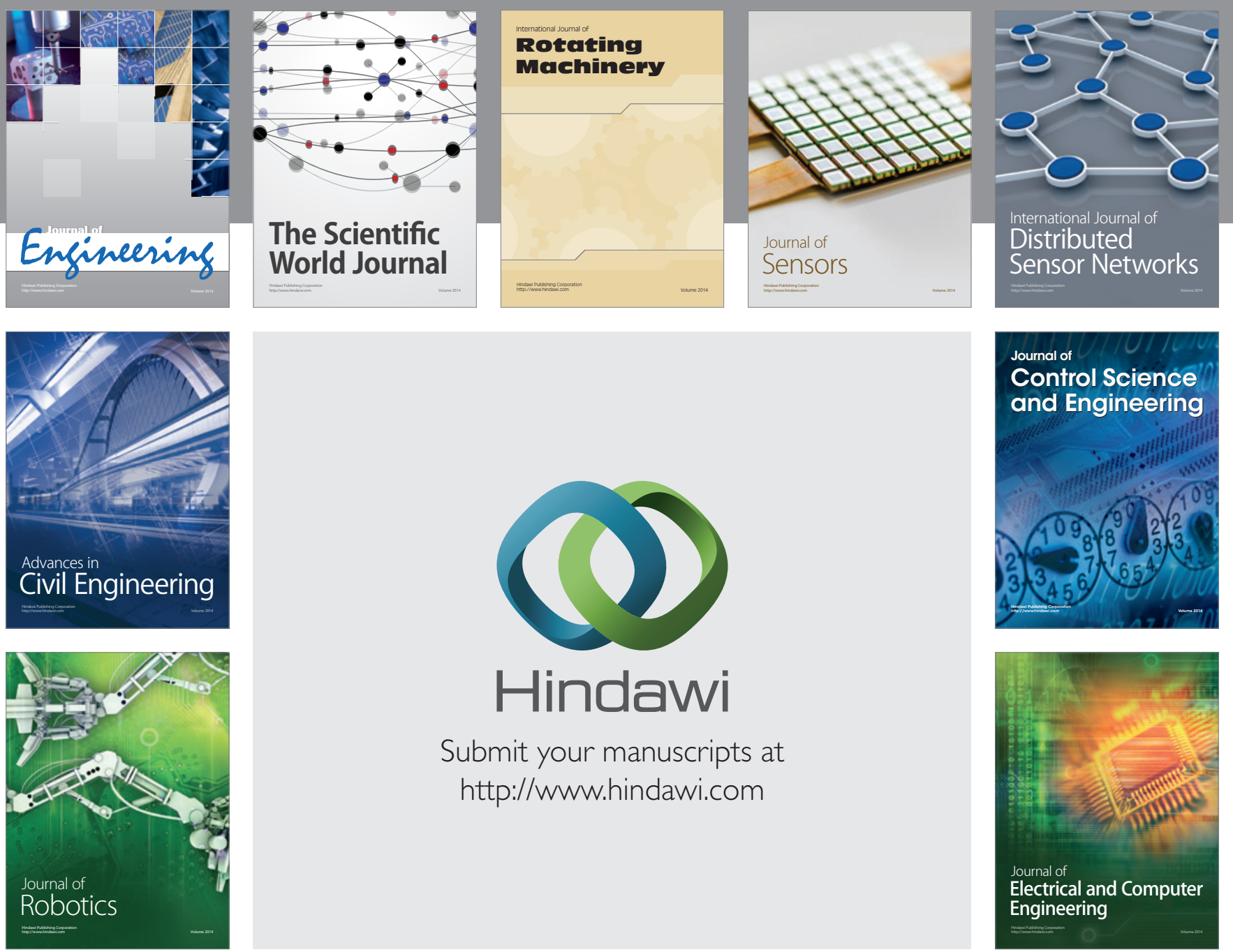

Submit your manuscripts at

http://www.hindawi.com
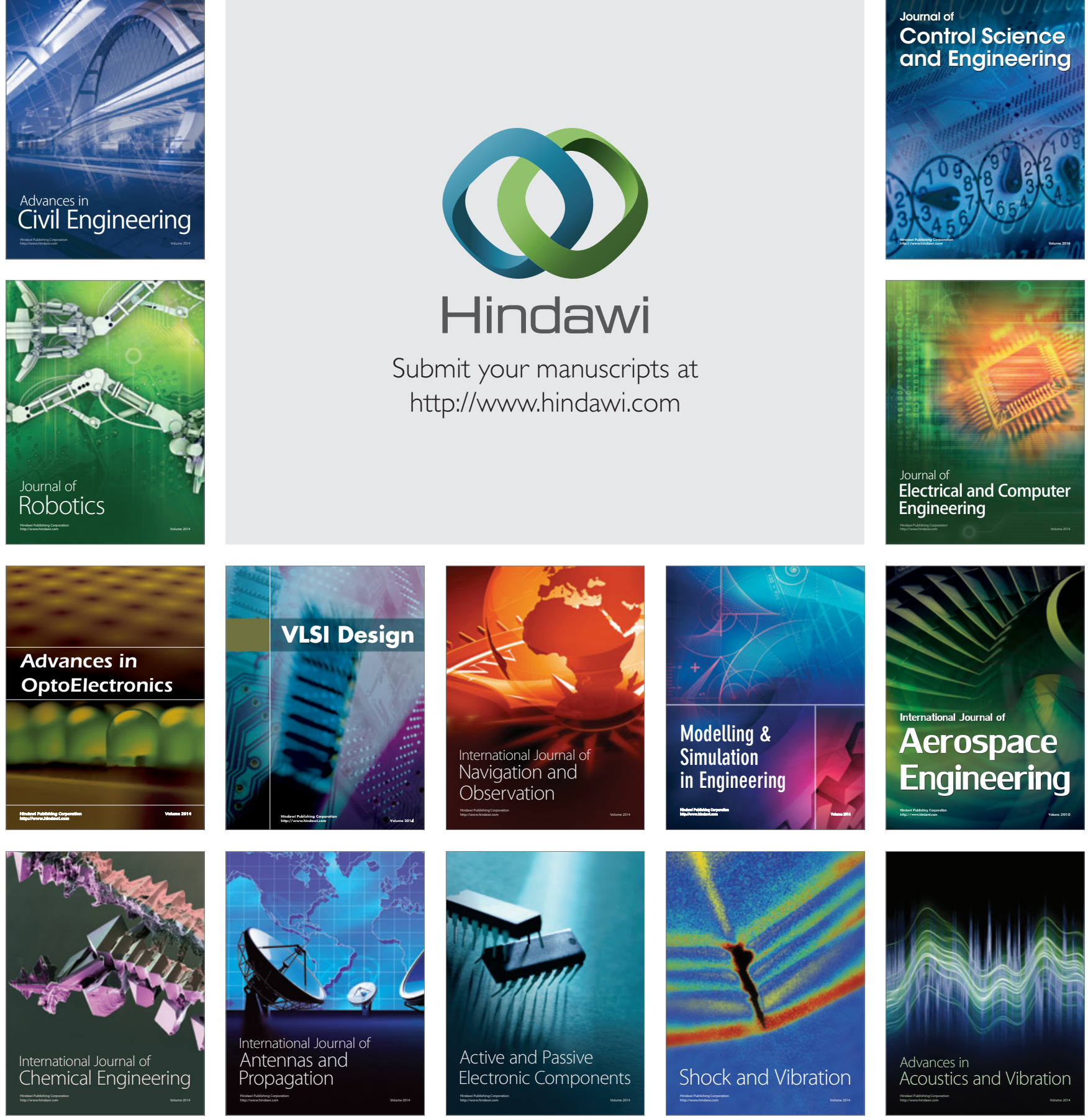